\title{
Epitaxial strain, metastable structure, and magnetic anisotropy in Co-based superlattices (invited)
}

\author{
Roy Clarke, S. Elagoz, W. Vavra, E. Schuler, and C. Uher \\ Department of Physics, The University of Michigan, Ann Arbor, Michigan 48109-1120
}

We explore the relationship between interface structure and magnetic anisotropy in three

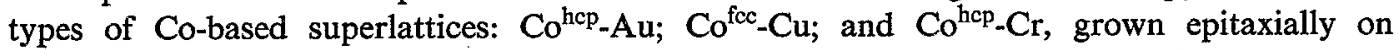
$\mathrm{GaAs}(110)$. For very thin layers of $\mathrm{Co}, \mathrm{Co}-\mathrm{Au}$, and $\mathrm{Co}-\mathrm{Cu}$ superlattices exhibit a perpendicular easy axis due to magnetoelastic contributions to the anisotropy energy. 'The magnetic anisotropy in $\mathrm{Co}-\mathrm{Cr}$ is found to be strongly dependent on growth conditions.

At slow deposition rates of $\mathrm{Co}$ the interface between $\mathrm{Co}$ and $\mathrm{Cr}$ becomes diffuse as is evidenced by a low saturation moment and a shift toward perpendicular anisotropy whereas samples with abrupt interfaces show predominantly parallel anisotropy. The $\mathrm{Cr}$ layers grow in a metastable hcp phase which appears to be paramagnetic. The results illustrate the influence of the heterointerface on magnetic properties.

\section{INTRODUCTION}

The realization of advanced techniques for ultrahighvacuum deposition of novel magnetic materials has inspired many studies in recent years. ${ }^{1,2}$ A key point of interest is that substrate-overlayer interactions can lead to considerable levels of interfacial strain and to dramatic modification of the thin-film structure. ${ }^{3}$ In some cases it is possible, with judicious choice of substrate orientation and appropriate buffer layers, to prepare metastable phases of magnetic elements. ${ }^{4,5}$

There is currently a great deal of activity in attempts to better understand the relationship between interfacial structure and the magnetic properties of ultrathin epitaxial layers. One important question relates to the role of elastic strain in influencing magnetic anisotropy, ${ }^{6}$ particularly for extremely thin magnetic layers. This is the regime in which a tendency has been observed for the easy axis of spin alignment to be perpendicular to the layers. ${ }^{7-10}$ It is also the limit at which the structure can support maximal elastic strains, i.e., where the layers do not greatly exceed the critical thickness for misfit dislocation strain relief. ${ }^{11}$

In this paper we present an overview of our recent results on Co-based epitaxial superlattices, emphasizing the role of interfacial strain and the appearance of metastable crystal structures. Co is a particularly interesting choice in this context since the structural stability of its bulk phases is known to be intimately related to its magnetic properties. ${ }^{12} \mathrm{We}$ compare the magnetic behavior of three different cobalt-based superlattice structures. In the first, $\mathrm{Co}-\mathrm{Au}$, the Co layers have the normal hep structure, albeit in a highly strained form. A second set of superlattices, $\mathrm{Co}-\mathrm{Cu}$, exhibits coherent fcc stacking of Co layers allowing us to probe the relative sizes of the magnetocrystalline and magnetoelastic contributions to the magnetic anisotropy. These two types of superlattice, consisting of alternate magnetic and nonmagnetic layers, are then contrasted with a third configuration: one in which cobalt is interleaved with $\mathrm{Cr}$. In this case a coherent metastable structure is observed with both Co and Cr layers stacked in a hcp arrangement. The results illustrate the importance of structural symmetry and lattice strain in influencing the mag- netic anisotropy. In particular, the role of the heterostructure interfaces is central to understanding these epitaxial magnetic films.

\section{SAMPLE PREPARATION}

The superlattice samples in this study were prepared in ultrahigh vacuum using a Vacuum Generators V-80M molecular-beam epitaxy (MBE) system with a background pressure better than $10^{-10} \mathrm{mbar}$. In each series of samples the Co layer thickness was varied from approximately 5 to $40 \AA$ while the $\mathrm{Au}, \mathrm{Cu}$, and $\mathrm{Cr}$ thicknesses were held fixed at 16,20 , and $10 \AA$, respectively. In addition, a limited number of samples have been prepared in which the effects of changing the $\mathrm{Au}, \mathrm{Co}$, or $\mathrm{Cr}$ "spacer" layers were investigated. The total superlattice thicknesses were typically $1500 \AA$.

Co was deposited from an electron-beam hearth at a rate of $0.2-0.4 \AA / \mathrm{s}$, while $\mathrm{Au}$ and $\mathrm{Cu}$ were evaporated using Knudsen cells giving a flux of $\sim 0.1$ and $\sim 0.2 \AA / \mathrm{s}$, respectively. In the case of $\mathrm{Cr}$ we used a high-temperature Knudsen cell operating at $\sim 1500^{\circ} \mathrm{C}$ with a deposition rate of $0.21 \AA / \mathrm{s}$. We found that the crystallographic quality of the superlattice layers in $\mathrm{Co}-\mathrm{Cr}$ superlattices improved somewhat as the rate of deposition of Co increased (see Fig. 1). This could be an indication of alloying between Co and $\mathrm{Cr}$, a complication with this particular superlattice system which we discuss in more detail below. Note that $\mathrm{Co}$ is practically immiscible with respect to both $\mathrm{Au}$ and $\mathrm{Cu}$ and so we do not expect, or observe, any alloying or interdiffusion problems in these cases. In fact, recent spinecho NMR probes show that the interfaces are atomically abrupt in our Co-Cu samples. ${ }^{13}$

The deposition of the superlattices starts with a cleaned and annealed (110) GaAs substrate. Usually, we first grow a 500-Å buffer layer of (110) Ge in order to provide a well-ordered, smooth substrate surface. Following the growth of the Ge buffer layer at $T \approx 550^{\circ} \mathrm{C}$, the substrate temperature is lowered to approximately $50^{\circ} \mathrm{C}$ for subsequent metal growth.

We have found that the deposition of a thin $(\sim 25 \AA)$ layer of $\mathrm{Co}$ on $\mathrm{Ge}$ (or GaAs directly) helps to bridge the 

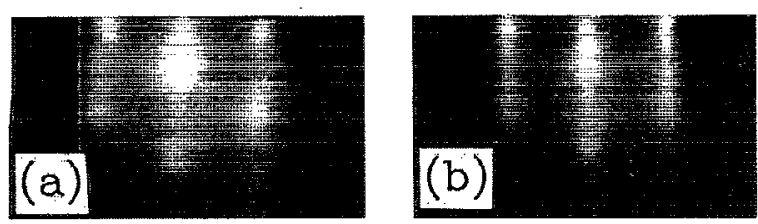

FIG. 1. Comparison of RHEED patterns for two superlattices of $20-\AA$ Co-10- $\AA \mathrm{Cr}$ grown at Co deposition rates of (a) 0.20 and (b) $0.35 \AA / \mathrm{s}$. The $\mathrm{Cr}$ deposition rate was fixed at $0.21 \AA \mathrm{A} / \mathrm{s}$ for both. Note the spotty arc pattern in (a) indicative of mosaic structure due to alloying.

(110) rectangular symmetry of the substrate and the (111) close-packed arrangement of the $\mathrm{Au}, \mathrm{Cu}$, or $\mathrm{Cr}$ layers. The bridging mechanism proceeds by means of the formation of a bcc phase ${ }^{5,14}$ of Co oriented in the (110) direction. With such a bridging layer we are able to grow high-quality superlattices of Co (hcp phase) and Au or Cr (also hep as discussed below).

For the Co-Cu superlattices it is necessary to employ two bridging layers between the Ge buffer and the actual superlattice layers. The first bridging layer is bcc $\mathrm{Co}$ as in the case above; this is followed by the growth of a layer of $\mathrm{Cu}$ where the first $60 \AA$ or so is in a distorted bec phase, and it then proceeds to grow fcc (111). Subsequent growth of Co on the (111) Cu surface leads to a cubic (fcc) metastable stacking arrangement. ${ }^{15}$ The growth techniques outlined here are described in detail elsewhere ${ }^{16}$ but it is worthwhile to note the importance of epitaxial bridging structures in implementing growth schemes for high-quality single-crystal superlattices based on Co.

\section{STRUCTURAL CHARACTERIZATION}

Reflective high-energy electron diffraction (RHEED) was used as an in situ probe of the layer structure and orientation during growth. In most cases well-defined RHEED patterns were obtained on the uppermost surfaces of the as-grown superlattices indicating coherent epitaxial growth. More detailed structural measurements were performed using $\mathrm{x}$-ray scattering after the samples were removed from the MBE system. A 20- $\AA$ Au cap layer was used in all cases to protect the samples from surface oxidation. X-ray scans were carried out on a four-circle diffractometer both with the diffraction vector normal to the layers ("out-of-plane" scan) and parallel to the layers ("in-plane" scan). In this way we could characterize the layering quality, the sharpness of the interfaces, the uniformity of growth, and the in-plane epitaxial arrangements (see Fig. 2). Additional types of $\mathrm{x}$-ray scans, e.g., where the diffraction vector is inclined to the layers but its tip follows a trajectory normal to the layers ( $c^{*}$ scan), were used to investigate the stacking of the layers. ${ }^{15}$ This latter type of scan is especially useful for identifying the metastable phases which appear as the result of epitaxy.

In the bulk, $\mathrm{Co}$ and $\mathrm{Cr}$ are known to form an hcp alloy and so it is important to verify that our samples are indeed layered. This is achieved by performing an out-of-plane $\mathrm{x}$-ray scan. Since the atomic numbers of $\mathrm{Co}$ and $\mathrm{Cr}$ differ

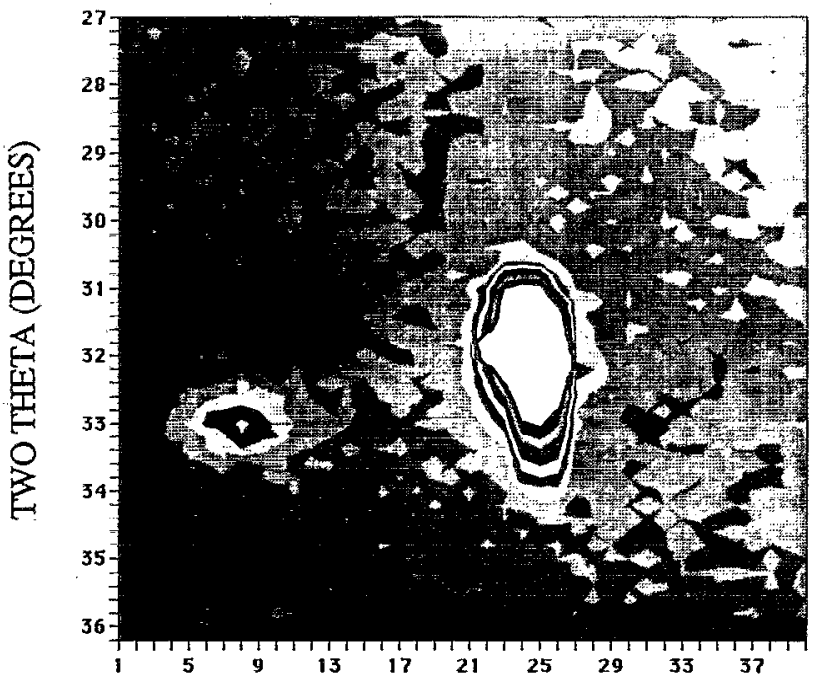

CHI (DEGREES)

FIG. 2. In-plane $\mathrm{x}$-ray scattering contour map of hep $\mathrm{Co}-\mathrm{Cr}$ superlattice showing epitaxial relationship of metal overlayer [weak (1120) peak] to GaAs substrate (intense peak at $2 \theta \approx 32^{\circ}$ ). The intensity contours are spaced at logarithmic intervals.

by only 3 , their scattering factors will be similar and we do not expect to observe many low-angle satellite peaks. ${ }^{17}$ However, several orders of satellites are evident in all samples and we have been able to fit the low-angle $x$-ray reflectivity curve (see Fig. 3) with a model in which the interfaces are step-like at the monolayer level. We are confident, therefore, that alloying is not a serious problem in this system provided that the growth rate for $\mathrm{Co}$ is greater than $\sim 0.3 \AA / \mathrm{s}$.

With the application of high-resolution $x$-ray diffractometry we have been able to characterize quantitatively the detailed structures of the superlattices including the degree of strain in each type of layer. Such measurements provide important clues towards understanding the metastable na-

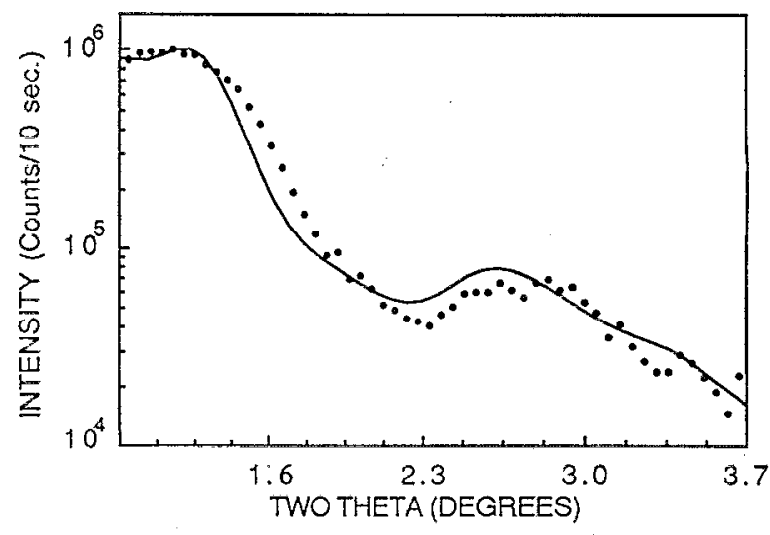

FIG. 3. Low-angle $x$-ray scattering data for the (18- $\AA$ Co-10- $\AA$ Cr $)_{54}$ superlattice (Co growth rate $0.35 \AA / \mathrm{s}$ ). The solid line is a fit calculated from a step model with no interface alloying. 

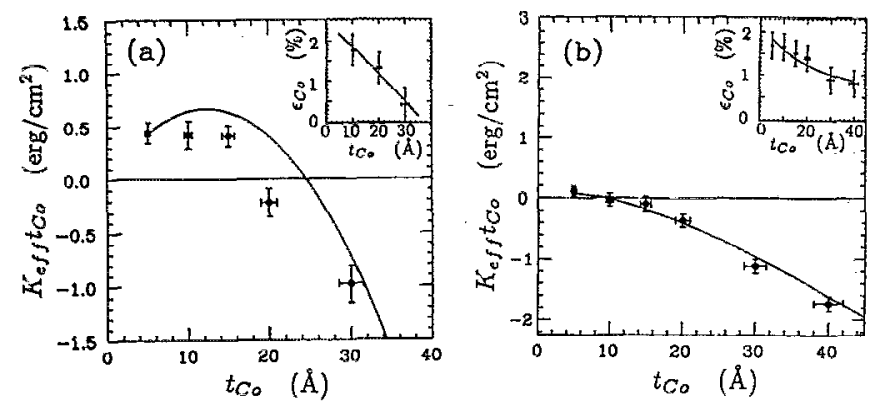

FIG. 4. Ambient-temperature magnetic anisotropy behavior as a function of thickness of Co layers, $t_{\mathrm{Co}}$, for (a) Co-Au superlattices with $16-\AA \mathrm{Au}$ spacer layers and (b) $\mathrm{Co}$-Cu superlattices with $20-\hat{A} \mathrm{Cu}$ spacer layers. The solid lines are fits to Eq. (3). Results from Ref. 10.

ture of these structures and the origin of the magnetic anisotropy.

\section{RESULTS AND DISCUSSION}

Magnetic measurements were carried out in a Quantum Design superconducting quantum interference device (SQUID) magnetometer. Hysteresis loops, measured with applied fields of up to $50 \mathrm{kOe}$, provided information on the effective magnetic anisotropy $K_{\text {eff }}$ through the relation

$$
K_{\mathrm{eff}}=\frac{1}{V} \int_{0}^{H_{\mathrm{sat}}}\left(M^{\perp}-M^{\|}\right) d H
$$

where $V$ is the total cobalt volume, and $M^{1}$ and $M^{\|}$are the magnetic moments with $H$ applied perpendicular and parallel to the layers. $K_{\text {eff }}$ is positive when the magnetization is preferentially oriented perpendicular to the film.

\section{A. Co-Au and Co-Cu superlattices}

We first discuss the results for $\mathrm{Co}-\mathrm{Au}$ and $\mathrm{Co}-\mathrm{Cu}$ superlattices. For all but the thinnest cobalt layers $(\sim 5 \AA$. the measured saturation moment is within $5 \%$ of the bulk value for $\mathrm{Co}$, confirming that we have negligible intermixing at the interfaces. ${ }^{10}$ The behavior of $K_{\text {eff }}$ vs Co thickness is compared in Figs. 4(a) and 4(b) for Co-Au and $\mathrm{Co}-\mathrm{Cu}$, respectively. Note that it is usual to plot $K_{\text {eff }} t_{\mathrm{Co}}$ rather than $K_{\text {eff }}$ so that the empirical relation

$$
K_{\mathrm{eff}}=\frac{2 K_{s}}{t_{\mathrm{Co}}}+K_{v}
$$

can be used to determine volume $\left(K_{v}\right)$ and surface $\left(K_{s}\right)$ magnetic anisotropy contributions from the slope and intercept of the plot. In this and previous work ${ }^{10}$ on this topic we have taken a different approach in analyzing $K_{\text {eff }}$ since the interpretation of "volume" and "surface" effects become somewhat blurred in a system composed mainly of interfaces.

Referring to Fig. 4, there is evidently a crossover from a parallel to a perpendicular easy axis as the Co layers are reduced in thickness. For $\mathrm{Co}-\mathrm{Au}$ this occurs at approximately $20 \AA$, at ambient temperature, and at $\sim 10 \AA$ for
$\mathrm{Co}-\mathrm{Cu}$. Following Chappert and Bruno, ${ }^{6}$ we have analyzed this behavior in terms of an effective anisotropy which has three contributions:

$$
K_{\mathrm{eff}}=K_{\mathrm{MC}}+K_{D}+K_{\mathrm{ME}}\left(t_{\mathrm{Co}}\right),
$$

where $K_{\mathrm{MC}}$ is the magnetocrystalline anisotropy (nominally taken as the bulk value of $5.56 \times 10^{6} \mathrm{erg} / \mathrm{cm}^{3}$ for hcp $\mathrm{Co}), K_{D}$ is the demagnetization term $\left(-2 \pi M_{s}^{2}\right)$ and $K_{\mathrm{ME}}\left(t_{\mathrm{Co}}\right)$ is a thickness-dependent magnetoelastic contribution:

$$
K_{\mathrm{ME}}=-\left(c_{11}+c_{12}-\frac{2 c_{13}^{2}}{c_{33}}\right)\left(\lambda_{A}+\lambda_{B}\right) \epsilon\left(t_{\mathrm{Co}}\right)
$$

The solid curves in Figs. 4(a) and 4(b) are fits to Eq. (3). Note that the saturation seen at the lower Co thicknesses is reproduced without invoking interface defects and other sample growth problems, as some authors have done. ${ }^{18}$ In fact, we have indisputable evidence for these systems that the interfaces are atomically sharp. ${ }^{13}$

The fit to the Co-Au data is based on the bulk hop values for $K_{\mathrm{MC}}$; also $c_{i j}$ are the elastic constants, and $\lambda_{A}$, $\lambda_{B}$ are the magnetostriction constants, for bulk hcp Co. The lattice strain $\epsilon\left(t_{C_{0}}\right)$ is measured from in-plane x-ray scans (see insets in Fig. 4) and decreases inversely as $t_{\mathrm{CO}}$, which is expected as the strained layers exceed the critical thickness. ${ }^{11}$

The fit to the anisotropy data for the $\mathrm{Co}-\mathrm{Cu}$ case (metastable fcc Co layers) provides an interesting comparison. In order to reproduce the observed thickness dependence it is necessary to use a value of $K_{\mathrm{MC}}$ which is only $20 \%$ of the bulk hcp value. The magnetoelastic coefficient is found to be essentially unchanged. This drastic reduction in $K_{\mathrm{MC}}$ is a direct result of the cubic symmetry of the metastable fcc phase compared to the uniaxial anisotropy of the hop phase. A similar difference has been observed at the hcp-fcc phase transition in bulk Co on heating to $400^{\circ} \mathrm{C}^{19}$

If instead of keeping the spacer layer fixed and varying $t_{\text {Co }}$ we now hold the ferromagnetic layer thickness constant while varying the separation of these layers; in this way we can investigate the effects of interlayer coupling. The behavior of the $\mathrm{Co}-\mathrm{Cu}$ system is interesting in this regard because there is some evidence for an oscillatory exchange interaction. ${ }^{20}$ Recent work suggests that for some thicknesses of $\mathrm{Cu}$ for example, an antiferromagnetic coupling exists between neighboring Co layers. ${ }^{21}$ Figure 5 shows the anomalous magnetic behavior resulting from a modification of the interlayer coupling. In this figure we show the variation in magnetic anisotropy with $t_{\mathrm{Cu}}$. One can clearly discern a more complex behavior than that found with varying $t_{\mathrm{Co}}$ [see Fig. 4(b)]. In particular we find evidence for oscillatory behavior with a period of $\sim 12 \AA$, a typical length scale that has been seen in other coupled ferromagnetic layer systems. The origin of this length scale, and the nature of the coupling itself, are currently under intense debate. An interesting conclusion relating to the results shown in Fig. 5 is that the magnetic anisotropy can be tailored by adjusting the nonmagnetic spacer layer. Moreover, the results suggest that for particular spacings it 


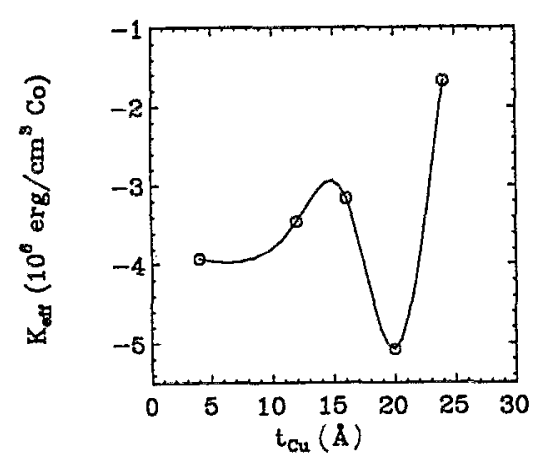

FIG. 5. Ambient-temperature magnetic anisotropy behavior of $\mathrm{Co}^{\text {foc }}-\mathrm{Cu}$ superlattices with fixed $20-\AA$ Co layers, as a function of Cu spacer layer thickness, $t_{\mathrm{Cu}}$. The solid lines are guides for the eye.

might be possible to significantly enhance the perpendicular spin orientation. Further experiments at thinner Co layers than the $20 \AA$ chosen here are required to verify this suggestion.

\section{B. Co-Cr superlattices}

The epitaxy of metastable phases of $\mathrm{Cr}$ was explored some years ago by Durbin et al. . $^{22}$ who reported an fcc phase of $\mathrm{Cr}$ grown on $\mathrm{Au}(100)$. In the present work we have shown that it is possible to stabilize an hcp phase of $\mathrm{Cr}$ in a $\mathrm{Co}-\mathrm{Cr}$ superlattice.

Figure 6 shows the dramatically different magnetic anisotropy behavior of two sets of $\mathrm{Co}-\mathrm{Cr}$ superlattices grown at different Co deposition rates, as described in Sec. II. The slower deposition rates probably result in $\mathrm{Co} / \mathrm{Cr}$ alloying at the interfaces. In addition to the structural evidence above (see, e.g., Fig. 1), we can also infer some degree of alloying from measurements of the specific magnetization at different Co layer thicknesses. ${ }^{23}$ These studies show a reduction in magnetization of approximately $25 \%-30 \%$ for samples grown at the faster rate $(0.30-0.35 \AA / \mathrm{s}$ of Co) compared to as much as a $70 \%$ reduction for a Co growth rate of $0.2 \AA / \mathrm{s}$. The suppressed moment in the latter case,

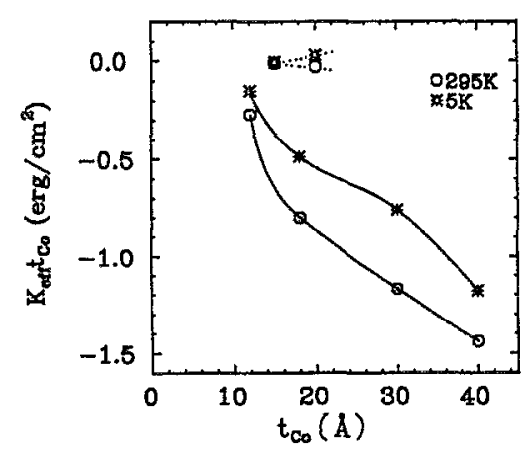

FIG. 6. Magnetic anisotropy behavior of $\mathrm{Co}-\mathrm{Cr}$ superlattices with fixed $10-\AA \mathrm{Cr}$ layers, as a function of Co layer thickness, $t_{\mathrm{C} \sigma}$. The data points connected by solid lines (guides for the eye) are for samples grown at the faster rates $(\sim 0.3-0.35 \AA \mathrm{A} / \mathrm{s})$. The uppermost data connected by dotted lines refer to samples grown at the slower rates $(\sim 0.2-0.25 \AA / \mathrm{s})$ where interface alloying is significant. along with a perpendicular anisotropy are both characteristics of Co-Cr alloys. ${ }^{24}$ The tendency toward perpendicular anisotropy in the alloyed samples is shown in Fig. 6 (dotted lines) along with the samples grown at the faster rate (solid lines). The strongly parallel anisotropy in the samples with abrupt interfaces was surprising however, since the $\mathrm{Cr}$ was expected ${ }^{25}$ to impose an in-plane tensile stress on the Co which would favor perpendicular anisotropy. Moreover, this relationship between the interface sharpness and the anisotropy in the Co-Cr system is interesting since it is just the opposite of that observed in other superlattice systems. Usually, a sharper interface will enhance the perpendicular anisotropy which is clearly not the case here.

Last we note that the saturation moments of the $\mathrm{Co}-\mathrm{Cr}$ samples with the sharpest interfaces (the samples with 12and $18-\AA$ Co layers) can be accounted for by assuming that the monolayer of $\mathrm{Co}$ atoms in contact with $\mathrm{Cr}$ at each interface have negligible moment and the rest of the Co retains its bulk moment. This yields moments with $4 \%$ of the measured values if the $\mathrm{Cr}$ is paramagnetic. This is of intercst in the context of a recent prediction, ${ }^{26}$ based on a generalized Stoner theory, that hcp $\mathrm{Cr}$ is close to being ferromagnetic.

In conclusion, the results presented here reveal an important connection between epitaxial structure and the behavior of the magnetic anisotropy in Co-based superlattices. In the comparison of $\mathrm{Co}^{\text {hep }}-\mathrm{Au}$ and $\mathrm{Co}^{\mathrm{fcc}}-\mathrm{Cu}$ superlattices, the larger magnetocrystalline energy of the hcp phase of Co favors a perpendicular easy axis but this is not sufficient to overcome the shape anisotropy until the magnetoelastic term dominates at small $t_{\mathrm{Co}}$ (where the lattice strain is significant). For $\mathrm{Co}^{\text {hcp }}-\mathrm{Cr}$, the $\mathrm{Cr}$ is stabilized in an hcp phase, and interfacial diffusion is growth-rate dependent. The $\mathrm{Co}-\mathrm{Cr}$ superlattices also exhibit an unusual relationship between interface sharpness and anisotropy in that the sharper the interface the more parallel the anisotropy becomes.

\section{ACKNOWLEDGMENT}

This work was supported in part by NSF Grant No. DMR 8805156.

\footnotetext{
'See, for example, Thin Film Growth Techniques for Low-dimensional Structures, edited by R. F. C. Farrow, S. S. P. Parkin, P. J. Dobson, J. H. Neave, and A. S. Arrott (Plenum, New York, 1987).

${ }^{2}$ Growth, Characterization and Properties of Ultrathin Magnetic Films and Multilayers, edited by B. T. Jonker, J. P. Heremans, and E.' E. Marinero, Materials Research Society Proceedings 151 (MRS, Pittsburgh, 1989).

${ }^{3}$ For a review see: L. M. Falicov et al., J. Mater. Res. 5, 1299 (1990).

${ }^{4}$ W. A. Jesser and J. W. Matthews, Philos. Mag. 17, 461 (1968); O. Haase, Z. Naturforsch. Teil A 14, 920 (1959); B. Heinrich, K. B. Urquhart, J. R. Dutcher, S. T. Purcell, J. F. Cochran, and A. S. Arrott, J. Appl. Phys. 63, 3863 (1988).

${ }^{5}$ G. A. Prinz, Phys. Rev. Lett. 54, 1051 (1985).

${ }^{6}$ C. Chappert and P. Bruno, J. Appl. Phys. 64, 5736 (1988).

${ }^{7}$ U. Gradmann, J. Magn. Magn. Mater. 54-57, 733 (1986).

${ }^{8}$ P. F. Carcia, A. D. Meinhaldt, and A. Suna, Appl. Phys. Lett. 47, 178 (1985).

${ }^{9}$ F. J. A. den Broeder, D. Kuiper, A. P. van de Mosselaer, and W. Hoving, Phys. Rev. Lett. 60, 2769 (1988).
} 
${ }^{10}$ C. H. Lee, H. He, F. J. Lamelas, W. Vavra, C. Uher, and R. Clarke, Phys. Rev. B 42, 1066 (1990).

"IJ. W. Matthews and A. E. Blakeslee, J. Cryst. Growth 27, 118 (1974).

${ }^{12}$ B. I. Min, T. Oguchi, and A. J. Freeman, Phys. Rev. B 33, 7852 (1986) P. M. Marcus and V. L. Moruzzi, Solid State Commun. 55, 971 (1985).

${ }^{13} \mathrm{~K}$. Le Dang, P. Veillet, H. Hui, F. J. Lamelas, C. H. Lee, and R. Clarke, Phys. Rev. B 41, 12, 902 (1990).

${ }^{14}$ R. Walmsley, J. Thomson, D. Friedman, R. M. White, and T. H. Geballe, IEEE Trans. Magn. MAG-19, 1992 (1983).

${ }^{15}$ F. J. Lamelas, C. H. Lee, H. Hui, W. Vavra, and R. Clarke, Phys. Rev. B 40, 5837 (1989).

${ }^{16}$ C. H. Lee, H. Hui, F. Lamelas, W. Vavra, C. Uher, and R. Clarke, Phys. Rev. Lett. 62, 653 (1989); H. Hui, Ph.D. thesis, The University of Michigan, 1990 (University Microfilms, Inc., Ann Arbor).

${ }^{17}$ F. J. Lamelas, H. D. He, and R. Clarke, Phys. Rev. B 43, 12296 (1991).

${ }^{18}$ H. J. G. Draaisma, W. J. M. de Jonge, and F. J. A. den Broeder, J. Magn. Magn. Mater. 66, 351 (1987).
${ }^{19}$ W. Sucksmith and J. E. Thompson, Proc. R. Soc. London Ser. A 225, 362 (1954).

${ }^{20}$ D. Pescia, D. Kerkmann, F. Schumann, and W. Gudat, Z. Phys. B 78, 475 (1990).

${ }^{21}$ A. Cebollada, J. L. Martinez, J. M. Gallego, J. J. de Miguel, R. Miranda, S. Ferrer, F. Batallan, G. Fillion, and J. P. Rebouillat, Phys. Rev. B 39, 9726 (1989); P. Bruno and C. Chappert, Phys. Rev. Lett. 67, 1602 (1991).

${ }^{22}$ S. M. Durbin, L. E. Berman, B. W. Batterman, M. B. Brodsky, and H. C. Hamaker, Phys. Rev. B 37, 6672 (1988).

${ }^{23}$ W. Vavra, S. Elagoz, R. Clarke, and C. Uher (to be published).

${ }^{24} \mathrm{~S}$. Iwasaki and R. Ouchi, IEEE Trans. Magn. MAG-14, 849 (1978).

${ }^{25} \mathrm{Here}$ we note that bcc $\mathrm{Cr}$ has a $2 \%$ larger lattice constant than bcc $\mathrm{Co}$ and we assume that this scaling can be applied to hcp $\mathrm{Cr}$ and $\mathrm{Co}$.

${ }^{26}$ D. A. Papaconstantopoulos, J. L. Fry, and N. E. Brener, Phys. Rev. B 39, 2526 (1989). 